\title{
Human Habitation in a Lunar Electric Rover During a 14-Day Field Trial
}

\author{
Harry Litaker, Jr. ${ }^{1}$, Shelby Thompson ${ }^{1}$, and Robert Howard, Jr. ${ }^{2}$ \\ ${ }^{1}$ Lockheed Martin, JSC, Houston, TX \\ ${ }^{2}$ Johnson Space Center, NASA
}

\begin{abstract}
Various military and commercial entities, as well as the National Aeronautics and Space Administration (NASA), have conducted space cabin confinement studies. However, after an extensive literature search, only one study was found using a simulated lunar rover (LUNEX II), under laboratory conditions, with a crew of two for an eighteen day lunar mission. Forty-three years later, NASA human factors engineers conducted a similar study using the Lunar Electric Rover (LER) in a dynamic real-world lunar simulation at the Black Point Lava Flow in Arizona. The objective of the study was to obtain human-in-the-loop performance data on the vehicle's interior volume with respect to human-system interfaces, crew accommodations, and habitation over a 14-day mission. Though part of a larger study including 212 overall operational elements, this paper will discuss only the performance of fifty different daily habitational elements within the confines of the vehicle carried out by two male subjects. Objective timing data and subjective questionnaire data were collected. Results indicate, much like the LUNEX II study, the LER field study suggest that a crew of two was able to maintain a satisfactory performance of tasks throughout the 14-day field trail within a relative small vehicle volume.
\end{abstract}

\section{INTRODUCTION}

Habitation is the space which determines the overall living and operational environment for an individual within a space vehicle which affects the quality of daily life and productivity onboard the vehicle (Celentano et al., 1963; Bond \& Campbell, 1995). NASA's Gemini VII study was the first real-world 14-day habitation study in space. NASA (1966) concluded "with a well-prepared crew within the confines of the spacecraft" a crew of two could live and work efficiently in space. The only lunar rover habitat study was conducted under the Apollo Logistics Support System (ALSS) in 1966, under laboratory conditions, inside a lunar rover simulator designated the LUNEX II for 18-days (Haaland et al., 1966). By measuring human performance with the cabin's volume, investigators conclude the crew was able to maintain satisfactory performance throughout the 18day simulation. Forty-three years later, NASA human factor engineers examined human performance within the Lunar Electric Rover (LER) over a 14-day simulated lunar mission in a dynamic real-world environment. As with Gemini VII and LUNEX II, the purpose of the field trail was to obtain human performance data on the volume of the vehicle's configuration with a crew of two.

\section{METHODS}

\section{Subjects}

Two male participants, one being a flight experienced astronaut and one being a professional geologist, operated and interfaced with all the internal and external systems onboard LER while on a 14-day simulated lunar mission. In preparation of the desert trials, they took part in several dry run tests at the Johnson Space Center's (JSC) Rock Yard to become familiar with all the vehicle's internal and external systems.

\section{Environment}

The test environment for the trial occurred at the Black Point Lava Flow; approximately 40 miles north of Flagstaff, Arizona. Having a wide variety of geologically relevant surface features, the test site presents many opportunities to evaluate human performance with both the Intravehicular Activities (IVA) and Extravehicular Activities (EVA) capabilities of the LER. Surface characteristics included slopes with an approximate $6^{\circ}$ of vertical from top to bottom, soil mechanics ranging from lose grain to hard-packed, surface properties ranging from flat/smooth to rocky, and some minimal vegetation.

\section{Equipment}

The LER is a high fidelity functional vehicle which provides the crew a safe haven from the hazardous environment of the lunar surface, a living area for multiple day missions away from the lunar outpost, and a rapid EVA deployment system for scientific exploration of the surface (see Figure 1). Total interior volume of the vehicle was calculated at 10.8 cubic meters with a net habitable volume (NHV) of approximately 8.6 cubic meters, resulting in about $79 \%$ functional volume. Net habitable volume is defined as the total remaining volume available to crew after accounting for the loss of volume due to equipment, stowage, and any other structural inefficiencies which decrease functional volume.

\section{Procedures}

A traverse plan was developed to identify and prioritize specific sites of scientific interest using remote sensing data that is of equivalent resolution to that expected of a crewed lunar mission without preceding robotic or crewed missions to that site. Other operational tasks were developed to 
exercise all the vehicle's human-machine interfaces. Target sites along the fourteen day traverse path were added or removed on-the-fly to reprioritized and optimize crew efficiency, thus providing a more realistic mission testing environment.

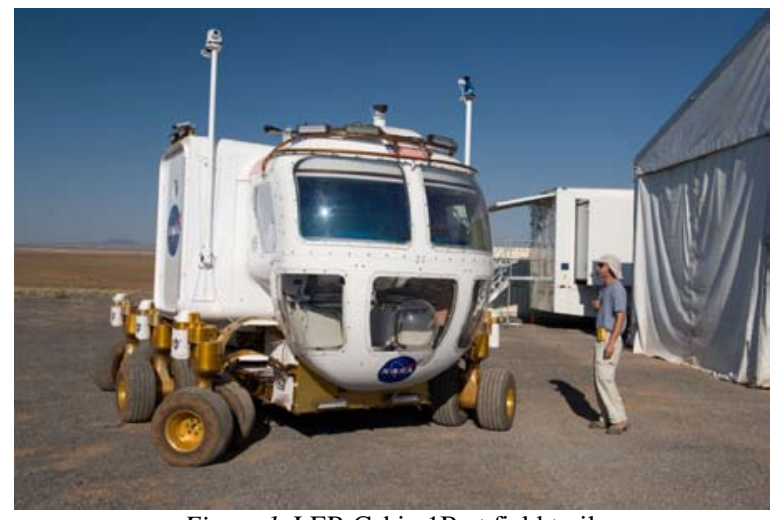

Figure 1. LER Cabin 1B at field trail.

A combination of human factors metrics and customized post-questionnaires were used to evaluate the LER and human performance. Some metrics were recorded by the subjects on specific days. Detailed field notes of all planned and unplanned traverse activities for that day were collected daily as well. Each scale represents a different human aspect; thus, when taken in combination, it could assist in developing a complete human performance assessment for a particular task or set of tasks. By pre-test definition, a rating of $\leq 4$ indicates the habitation element was acceptable (Kosmo et al., 2009). Though the field trial examined the LER vehicle in 212 operational elements, this paper will only discuss the habitation of two crew within the rover over the 14-day mission.

\section{RESULTS}

During the 14-day field trial, the crew recorded 316 hours and 55 minutes in total mission time. Mission time officially started at 9:59:00 on 31 August 2009 and ended at 15:00:00 on 13 September 2009. This time includes operation of the vehicle, EVA time, translation time, maintenance time, and contextual observation time. A breakdown of time data by elements was conducted to understand crew productivity (see Table 1).

For the 14-day LER mission, human factor engineers examined a total of fifty different habitational elements within the vehicle which the crew performed on a daily basis. For ease of the reader, authors decided to separate the subjective habitation data into four different sections. Due to the small sample size $(\mathrm{N}=2)$, descriptive statistics using the Grand Mean (GM) will be used across the 14 days.
Table 1.

Field Times for the LER 14-Day Mission

\begin{tabular}{cc}
\hline Elements & Time (hh:mm:ss) \\
\hline LER Driving & $23: 27: 00$ \\
EVA & $7: 18: 00$ \\
On-Duty & $137: 56: 00$ \\
Off-Duty & $178: 59: 00$ \\
Rescue & $23: 26: 00$ \\
Total Habitat & $285: 36: 30$ \\
\hline
\end{tabular}

\section{Daily Food Prep, Hygiene, and WCS Operations}

This first section discusses fourteen habitation elements. Of the fourteen studied, the crew rated volume to limit crosscontamination, WCS privacy, frequency of trash/waste removal using the Suit Port Transfer Module (SPTM), accessibility to the water dispenser, housecleaning, and volume for preparing and eating a meal within the vehicle as acceptable (see Table 2). As per the LER Concept of Operations (ConOps), trash was to be taken out every third day. The crew reported this worked well noting that trash tended to build up quickly over 3-day period and the odor started to become unpleasant.

Seven elements were considered as borderline by the crew while one was considered totally unacceptable. Access to food stowage did become an issue with the crew due to reconfiguring the aft cabin especially when it was a single person EVA. It became difficult for the IVA crewmember to access anything within the benches or floor panels. WCS operations had some issues, especially with deploying the unit when both benches were down. The crew reported there was not enough room with both benches down which made it uncomfortable. This also held true when both sleep stations are deployed with the aisle being approximately $0.55 \mathrm{~m}$ in width. As for the LER hygiene mist sprayer hose, the crew stated it was not needed in the vehicle. Their preference for personal hygiene was using a wet towel or sponge to take a bath.

Table 2.

Ratings for Food Prep, Hygiene, and WCS Operations

\begin{tabular}{lc}
\hline \multicolumn{1}{c}{ Elements } & $\boldsymbol{G M}$ \\
\hline Food Stowage & 4.4 \\
Meal Prep/Eating & 2.8 \\
Water Dispenser & 3.4 \\
Deploy/Stow WCS & 5.0 \\
Hygiene Shower & 5.0 \\
WCU Use & 5.8 \\
WCS Privacy & 3.9 \\
Waste/Trash Stowage & 5.9 \\
Odor of Waste/Trash & 8.5 \\
Vacuum for Trash & 4.9 \\
Effectiveness of Space Bags & 4.9 \\
Frequency of Trash Removal & 3.8 \\
Cross-Contamination & 4.4 \\
House Cleaning & 3.1 \\
\hline
\end{tabular}


Much like the 2008 field trial (Litaker et al., 2008), having a dedicated stowage area, trash management system, an odor control became the issues with odor control being rated totally unacceptable $(G M=8.5)$. Like other systems in the LER, the trash management system was redesigned to help correct these issues. The new system was to take the daily trash, put it into a medium sized Space Bag, and vacuum seal the bag at the end of each day. After collecting 3 days worth of trash, the crew would transfer the three medium Space Bags into one large Space Bag and vacuum seal the bag and transfer it out using the SPTM. First issue was the crew ended up putting three days of trash in one medium Space Bag. This caused one bag to break which the crew fixed by using 2-inch duct tape. They also noted the Space Bag did not control odor well and was heavy when it contained 3 days of trash.

\section{Internal Volume and Stowage}

For internal volume and stowage, seven elements were studied. Volume, accessibility, clearance, and overall design of the personal soft lockers were rated by the crew as totally acceptable. The crew reported the soft locker volume was great for a 14-day mission as well as the accessibility to the lockers (see Table 3). They liked the flipped down front flaps and locker depth to hold personal items. The crew liked the overall design and concept of the soft locker system; however, reconfiguration of the system became a major issue. Reconfiguration time was a factor with the crew observing approximately 5 minutes per reconfigure per EVA with 4 EVAs per day for 14 days times 4 crewmembers would equal 18 hours and 40 minutes per mission. With the crew having to relocate the lockers, space within the vehicle started to become cramped and crowded (see Figure 2).

Access to stowage items was rated as borderline by the crew $(G M=4.8)$. Comments on vehicle stowage stemmed from the reconfiguration issues previously discussed. The crew, however, did express bench stowage was the best use of available space within the vehicle.

Table 3.

Ratings for Internal Volume and Stowage

\begin{tabular}{lc}
\hline \multicolumn{1}{c}{ Elements } & GM \\
\hline Volume of Soft Locker & 2.8 \\
Access to Lower Hard Locker & 3.0 \\
Access to Upper Soft Locker & 2.6 \\
Clearance Seated at Soft Locker & 2.6 \\
Overall Design of Soft Locker & 2.9 \\
Location of Stowage & 4.4 \\
Access to Stowage & 4.8 \\
\hline
\end{tabular}

\section{Exercise}

The crew was able to use the exercise ergometer for twelve out of fourteen days for an hour per crewmember per day. Of the sixteen elements, ten were rated as acceptable. These acceptable elements included access to the exercise equipment within the LER, setup and stowage of the equipment, stability/adjustability of the seat, design of the ergometer for aerobic exercise in the LER and volume to perform both resistive and aerobic exercise in the LER (see Table 4).

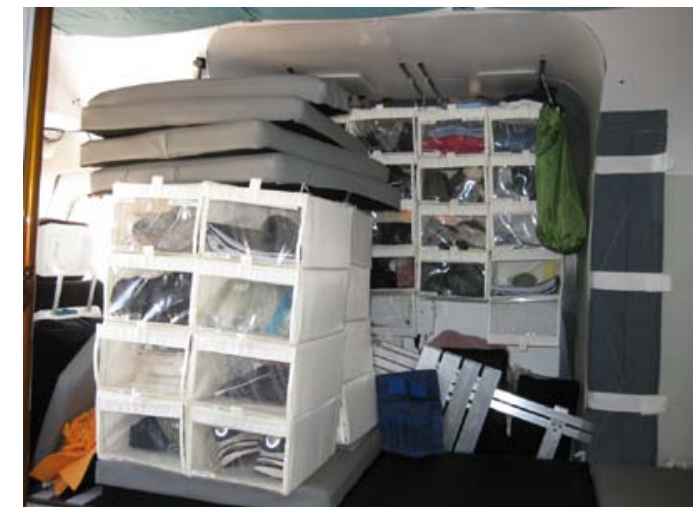

Figure 2. LER cabin after a reconfiguration for EVA.

Table 4 .

Ratings for Exercise Activities and Ergometer Design

\begin{tabular}{lc}
\hline \multicolumn{1}{c}{ Elements } & GM \\
\hline Access to Equipment & 3.3 \\
Setup of Equipment & 3.6 \\
Comfort of Seat & 5.3 \\
Stability of Seat & 3.4 \\
Adjustability of Seat & 3.5 \\
Volume for Resistive Exercise & 2.8 \\
Accessibility to other LER Stations during Resistive & 4.4 \\
Resistive Exercise while LER is Moving & 5.5 \\
Ergometer Design for Resistive Exercise & 4.9 \\
Volume for Aerobic Exercise & 3.3 \\
Accessibility to other LER Stations during Aerobic & 4.9 \\
Aerobic Exercise while LER is Moving & 6.1 \\
Ergometer Design for Aerobic Exercise & 3.4 \\
Ability to Stow Equipment & 3.5 \\
Stowage for Equipment & 3.6 \\
Overall Exercise in LER & 2.9 \\
\hline
\end{tabular}

The crew also reported the overall ability to exercise in the LER was acceptable. They came up with several ways to exercise within the vehicle in conjunction with using the ergometer. The LER provided plenty of space for doing such exercises as leg lunges, pushups, bench press with bands, and tricep dips. The interior volume was able to give the crew the ability to use a variety of exercise elements during their hour long session.

However, the crew did express some issues with the system. The ability of a crew to perform aerobic exercise while the vehicle was moving was the highest rated concern among the crewmembers $(G M=6.1)$. The crew reported it depended on the terrain in which the LER was traveling. If the terrain was flat and smooth, they did not have an issue with exercising; however, if the terrain was bumpy and rough, exercise was not possible. Just walking from the aft cabin to the cockpit of 
the LER while on rough terrain was difficult according to the crew. This also held true for resistive exercise as well (see Figure 3).

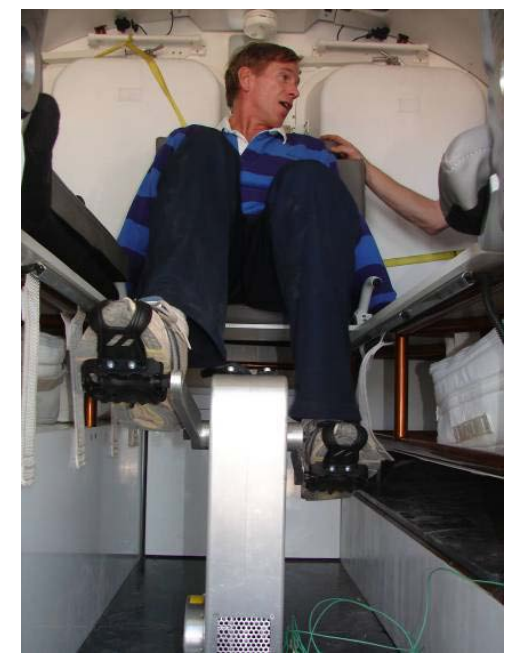

Figure 3. Crewmember doing aerobic exercise with ergometer. Note the spacing between the pedals and the aisle way.

Accessibility of the non-exercising crewmember to other stations within the vehicle while a crewmember was performing either aerobic or resistive exercise was also rated high. The crew indicated they tried to make sure certain items were stationed up in the cockpit area. With the ergometer in the aft section aisle way of the LER, the pedals of the device do not allow for any space to pass for the nonexercising crewmember. The crew also observed this limited aisle space as well during setup.

Seat comfort was reported at borderline by the crew. They reported the absence of any type of back support and would add several bench cushions to get comfy. As for the design of the ergometer itself, the crew reported several concerns. Ratings for the device's design were high with the crew complaining about the weight, pedals, and displays. The crew noted, several times during the trail, the weight. Noticing that for such a heavy piece of gear, the ergometer seemed fragile when it was being used. Pedals also became an annoyance to the crew. They reported the pedal attach/detach design was way too difficult to remove. The biggest observation on the pedal design was the fact the pedals, once locked on the device, was so tight the crew almost injured themselves taking them off. In fact, the crew stated they would leave one on just so they would not have to take the pedals on and off.

\section{Sleep Stations}

Generally, the crew reported the LER sleep stations as acceptable (see Table 5). Of the thirteen elements, the crew rated five - volume of station, layout, privacy, within station light quality, and personal item access—as totally acceptable.
They noted with the two soft lockers hanging in the side hatch and the high ceiling height, it gave them the perception of a spacious volume within the station (see Figure 4).

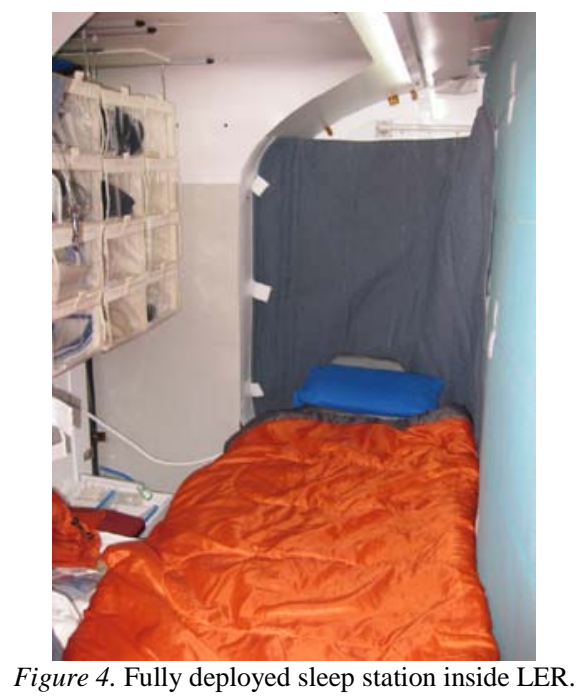

Egressing out of the station when the curtains were deployed was considered somewhat acceptable. The crew noted with the curtains down, there was limited access to the WCS and the noise from the Velcro was a concern for the other sleeping crewmember. As for the sleep quality, the crew stated they like the large side curtain for its sound and light dampening qualities which made sleeping a lot quieter. However, they reported the bench cushions by the front seats were awkward to lay on and some time was spent adjusting the pads for the right comfort. The LER's lighting controls were outside the sleep station which causes an issue. The crew reported there was no easy access to the controls.

Table 5.

\begin{tabular}{lc} 
Ratings for Sleep Station & \\
\hline \multicolumn{1}{c}{ Elements } & GM \\
\hline Curtain Deployment by 1 crew & 3.6 \\
Ease of Seat Reconfigure & 4.8 \\
Volume of Sleep Station & 1.9 \\
Sleep Station Layout & 2.1 \\
Volume of Personal Privacy & 2.3 \\
Sleep Quality & 3.8 \\
Light Quality in Sleep Station & 2.6 \\
Lighting Control & 4.4 \\
Access to Personal Items & 2.9 \\
Ease of Ingress into Station & 4.6 \\
Ease of Egress into Station & 4.1 \\
Curtain Stowage by 1 crew & 4.6 \\
Overall Design of Station & 3.3 \\
\hline
\end{tabular}

Ease of ingress, single crew curtain stowage, and seat reconfiguration were rated by the crew as borderline. They noted there was too much Velcro in the new design. Getting in and out of the station was not that easy without having to take most of the curtains apart. Curtain stowage by a single 
crewmember still remains an issue, especially with the large main curtain. Though the crew stated it was possible to accomplish; however, the weight and curtain support straps did not make it an easy task. Finally, the front seat reconfiguration reported the highest rating by the crew (GM $=4.8$ ). This was due to having to adjust the seat with two hands. Access to the adjusting tabs was difficult and getting the seats in the correct lateral placement was not optimal. As for the overall design, the crew rated the station as acceptable. Though needing some minor tweaking, the crew stated it was a huge part of the successful habitability of the LER for the two week mission.

\section{CONCLUSIONS}

The purpose of the desert trial was to obtain human performance data on the lunar electric rover with respect to crew accommodations and habitation to support a 14-day exploration lunar mission. Though with human factors data collected on only a crew of two, the following conclusions drawn are still valid concerns to future designs.

A dedicated trash area that was easily accessible for daily use and helps to control odor until disposal in needed. The aisle width of $0.55 \mathrm{~m}$ made WCS operations an issue with crew comfort. The soft locker system was deemed good by the crew. In fact, they indicated it was too much volume and suggested decreasing the volume by $50 \%$ for a 2 -week mission. Reconfiguration with this system became extremely time consuming for the crew and cabin volume started to decrease rapidly. The ergometer design showed several issues including the absence of back support for the seat, weight, pedal design and display design. A redesign of the seat to include back support; better attach/detach method of the pedals; improved readability for the display; and use oft with lighter weight materials and easier assembly are needed. With the general acceptance of the sleep station, improving the design consist of conformal custom curtains to block out more light on the sides, incorporate a small zippered section in the large curtain for easy egress and ingress, and using less ridged forward and aft curtains. The crew reported there would be no issues with a crew of two doing a 14-day mission without ever getting out of the LER. The crew noted a LER lunar mission would be more like a hiking trip where simple, lightweight, reusable items would be required for quick, easy access and consolidation.

Much like the LUNEX II study, the LER field studies are indicating the same results in performance of tasks for a crew of two in a relative small volume. By continuing a humancentered design approach and collaborating with designers to capture information in their design, the aim is to enhance human performance and comfort, while lending confidence to both future lunar habitation design, as well as planetary vehicle design.

\section{ACKNOWLEDGEMENTS}

We would like to recognize Dr. Michael Gernhardt for obtaining the funding for the project, Dr. Andrew Abercomby for his work on the engineering test plan, the Habitability Design Center at NASA/JSC: Rich Szabo, Carl Conlee, and Evan Twyford, for their illustrative and design work. Regan Geeseman at NASA/JSC Information Resources Directorate (IA) for his photographic work of the vehicle used throughout this paper. Thanks also to Dr. Robert Ambrose and his team at the NASA/JSC Automation, Robotics, and Simulation Division (ER), Extravehicular Activity Office (XA), and Joe Kosmo and Barbara Roming's team with the NASA/JSC Space Suit and Crew Survival System Branch (EC5) for all their hard work in designing, building, and testing this amazing vehicle.

\section{REFERENCES}

Bond, R. \& Campbell, P. (1995). Operational monitoring of MIR habitability. PIPS Database \#810530. Internal NASA Document.

Celentano, J.T., Amorelli, D., \& Freeman, G.G. (1963). Establishing a habitability index for space stations and planetary bases. American Institued of Aeronautics and Astronautics Manned Space Laboratory Conference, 2 May 1963, Los Angeles, CA, pp. 63-139.

Haaland, J.E., Grubbs, H.Y., Vaccaro, M. J., Graf, C.P., Kuechenmeister, T.J., Beigie, J.W., Kindead, R.D., Tuk, A.C., Stackhouse, S.P., \& Macek, F. (1966). Man system criteria for extraterrestrial roving vehicles phase $1 B-$ The LUNEX II simulation. Honeywell Interim Technical Report 12504-ITR2, MSFC NAS8-20006, Marshall Space Flight Center, Huntsville, AL.

Kosmo, J., Ambrose, R., \& Roming, B. (2009). Desert research and technology studies (D-RATS) September 2009 integrated field campaign test plan, 28 April 2009, National Aeronautics and Space Administration Lyndon B. Johnson Space Center, Houston, TX.

Litaker, Jr. H.L., Thompson, S., Howard, R., Szabo, R., Conlee, C. \& Twyford, E. (2008b). Small Pressurized Rover (SPR) three day desert trial: A human factors assessment. NASA/Johnson Space Center. Internal NASA Document, December 2008.

NASA (1966). Gemini program mission report of Gemini VII. NASA-TM-X-628892, N79-76319. National Aeronautics and Space Administration Manned Spacecraft Center, Houston, TX. 